\title{
Search for time-reversal-invariance violation in double polarized antiproton-deuteron scattering
}

\author{
Yuriy Uzikov ${ }^{1,2,3, \star}$ and Johann Haidenbauer ${ }^{4, \star \star}$ \\ ${ }^{1}$ Joint Institute for Nuclear Research, Dubna 141980, Russia \\ 2 Dubna State University, Dubna 141980, Russia \\ ${ }^{3}$ Department of Physics, M.V. Lomonosov Moscow State University, Moscow 119991, Russia \\ ${ }^{4}$ Institute for Advanced Simulation and Institut für Kernphysik, Forschungszentrum Jülich GmbH, D-52428 \\ Jülich, Germany
}

\begin{abstract}
.
Apart from the $p d$ reaction also the scattering of antiprotons with transversal polarization $p_{y}^{p}$ on deuterons with tensor polarization $P_{x z}$ provides a null-test signal for timereversal-invariance violating but parity conserving effects. Assuming that the timereversal-invariance violating $\bar{N} N$ interaction contains the same operator structure as the $N N$ interaction, we discuss the energy dependence of the null-test signal in $\bar{p} d$ scattering on the basis of a calculation within the spin-dependent Glauber theory at beam energies of 50-300 MeV.
\end{abstract}

\section{Introduction}

Under CPT symmetry time-reversal-invariance violating but parity conserving (TVPC) forces are considered as a possible source of $\mathrm{CP}$-invariance violation, which is required to account for the matterantimatter asymmetry in the universe [1]. In contrast to effects from time-reversal-invariance violation together with parity violation such as a permanent electric dipole moment (EDM) of elementary particles, so far much less attention was paid to TVPC effects. The reason why TVPC effects are interesting is that experimental limits on them are still rather weak, in particular, considerably weaker than those for the EDM.

Since the intensity of TVPC interactions within the standard model is extremely small [2], an observation of any effects at the present accuracy level of experiments would be a direct indication of physics beyond the standard model. Indeed a pertinent measurement is planned at the COSY accelerator in the Research Center in Jülich [3]. The observable in question is the integrated cross section for scattering of protons with transversal polarization $p_{y}^{p}$ on deuterons with tensor polarization $P_{x z}$. It provides a null-test signal for TVPC effects [4] and it will be measured in $p d$ scattering at $135 \mathrm{MeV}$ [3]. Theoretical studies of the energy dependence of the expected signal were performed at energies of the planned experiment [5-12] on the basis of the spin-dependent Glauber theory and demonstrate several unexpected effects. Among them are (i) the absense of the contribution from the

\footnotetext{
^e-mail: uzikov@jinr.ru

$\star \star$ e-mail: j.haidenbauer@fz-juelich.de
} 
lowest-mass meson-exchange ( $\rho$ meson) in the TVPC $N N$ interaction, caused by its specific isospin, spin and momentum dependence; (ii) a strong impact of the deuteron $D$-wave on the null-test signal due to a destructive interference between the $S$ - and $D$-wave contributions, even for zero transferred 3momentum; (iii) oscillating behaviour of the null-test signal as a function of the beam energy, i.e. the vanishing of the TVPC signal at some specific energies is possible even when the TVPC interaction itself is nonzero; (iv) a very small influence of the Coulomb interaction on the TVPC term of the $p d$ forward scattering amplitude $\widetilde{g}$. Furthermore, certain relations between differential observables of elastic $p d$ scattering caused by time-reversal-invariance requirements were obtained and the degree of their violation by TVPC $N N$ forces was studied $[13,14]$.

Since the spin structure of the amplitude for $p d$ - and $\bar{p} d$ elastic scattering is the same, it is obvious that the integrated cross section for scattering of a polarized $\left(p_{y}^{\bar{p}}\right)$ antiproton on tensor polarized $\left(P_{x z}\right)$ deuterons also provides a null-test signal for TVPC effects. Furthermore, the TVPC $\bar{N} N$ amplitude for elastic scattering contains the same operator structures as the one for TVPC $N N$ elastic scattering, except for the charge-exchange terms. Therefore, the formalism developed in Refs. [7, 8, 11] within the Glauber theory for the calculation of the null-test signal in $p d$ scattering can be straightforwardly applied to $\bar{p} d$ scattering too. However, due to differences in the hadronic part of the $p N$ and $\bar{p} N$ scattering amplitudes and also in the electromagnetic interactions, the energy dependence of the null test signal in $p d$ and $\bar{p} d$ interaction has to be different. In the present work the energy dependence of the null-test signal in $\bar{p} d$ scattering is studied on the basis of calculations within the spin-dependent Glauber theory using the spin-dependent $\bar{p} N$ amplitudes from a recent partial wave analysis of $\bar{p} p$ scattering [15].

\section{Null-test signal for time-reversal-invariance violation}

The total cross section for $\bar{p} d$ scattering with TVPC forces included can be written in the same form as for $p d$ scattering [7]

$$
\sigma_{t o t}=\sigma_{0}^{t}+\sigma_{1}^{t} \mathbf{p}^{\bar{p}} \cdot \mathbf{p}^{d}+\sigma_{2}^{t}\left(\mathbf{p}^{\bar{p}} \cdot \mathbf{m}\right)\left(\mathbf{p}^{d} \cdot \mathbf{m}\right)+\sigma_{3}^{t} P_{z z}+\widetilde{\sigma} p_{y}^{\bar{p}} P_{x z}^{d} .
$$

Here $\mathbf{p}^{\bar{p}}\left(\mathbf{p}^{d}\right)$ is the vector polarization of the initial antiproton (deuteron), $P_{z z}$ and $P_{x z}$ are the tensor polarizations of the deuteron, and $p_{y}^{\bar{p}}$ is the transversal component of the antiproton vector polarization. The $\mathrm{OZ}$ axis is directed along the beam direction $\mathbf{m}$, the $\mathrm{OY}$ axis is directed along the vector polarization of the antiproton beam $\mathbf{p}^{\bar{p}}$ and the $\mathrm{OX}$ axis is chosen to form a right-handed reference frame. The integrated cross sections $\sigma_{i}^{t}(i=0,1,2,3)$ are those which arise from a standard timereversal invariant and parity conserving interaction, while the last term $\widetilde{\sigma}$ appears only in the presence of the TVPC interactions and constitutes the TVPC null-test signal. The result (1) can be derived using phenomenological $\bar{p} d$ forward scattering amplitudes and the generalized optical theorem.

The evaluation of the integrated cross sections $\sigma_{i}^{t}$ and $\widetilde{\sigma}$ at beam energies $>100 \mathrm{MeV}$ can be done on the basis of the spin-dependent Glauber theory of $\bar{p} d$ scattering which is formulated similarly to the theory of $p d$ scattering given in Ref. [16]. Indeed, as shown in Ref. [17], this theory allows one to describe rather well available data on differential spin observables of $p d$ scattering in the forward hemisphere at beam energies of $135-200 \mathrm{MeV}$. For the antiproton-deuteron scattering this theory can be applied at even lower energies due to the presence of strong annihilation effects. In the Glauber theory one uses the elastic (on-shell) $\bar{N} N$ scattering amplitudes as input. Hadronic amplitudes of the $\bar{p} N$ scattering are taken here in the same form as for $p N$ scattering [16]

$$
\begin{array}{r}
M_{N}\left(\mathbf{p}, \mathbf{q} ; \boldsymbol{\sigma}, \sigma_{N}\right)=A_{N}+C_{N} \sigma \hat{\mathbf{n}}+C_{N}^{\prime} \sigma_{N} \hat{\mathbf{n}}+B_{N}(\sigma \hat{\mathbf{k}})\left(\sigma_{N} \hat{\mathbf{k}}\right)+ \\
+\left(G_{N}+H_{N}\right)(\sigma \hat{\mathbf{q}})\left(\sigma_{N} \hat{\mathbf{q}}\right)+\left(G_{N}-H_{N}\right)(\sigma \hat{\mathbf{n}})\left(\sigma_{N} \hat{\mathbf{n}}\right),
\end{array}
$$


where $\hat{\mathbf{q}}, \hat{\mathbf{k}}$ and $\hat{\mathbf{n}}$ are defined as unit vectors along the vectors $\mathbf{q}=\left(\mathbf{p}-\mathbf{p}^{\prime}\right), \mathbf{k}=\left(\mathbf{p}+\mathbf{p}^{\prime}\right)$ and $\mathbf{n}=[\mathbf{k} \times \mathbf{q}]$, respectively; $\mathbf{p}\left(\mathbf{p}^{\prime}\right)$ is the initial (final) antiproton momentum.

In general, the TVPC $N N$ interaction contains 18 different terms [18]. In the case of the onshell $N N$ scattering amplitude there are only three terms with different (independent) spin-momentum structures. In the present study we consider the following two terms for the TVPC (on-shell) $t$-matrix of elastic $\bar{p} N$ scattering which have the same structure as those in TVPC $p N$ scattering

$$
\begin{array}{r}
t_{\bar{p} N}=h_{N}\left[(\boldsymbol{\sigma} \cdot \mathbf{k})\left(\boldsymbol{\sigma}_{N} \cdot \mathbf{q}\right)+\left(\boldsymbol{\sigma}_{N} \cdot \mathbf{k}\right)(\boldsymbol{\sigma} \cdot \mathbf{q})-\frac{2}{3}\left(\boldsymbol{\sigma}_{N} \cdot \boldsymbol{\sigma}\right)(\mathbf{k} \cdot \mathbf{q})\right] / m_{p}^{2}+ \\
+g_{N}\left[\boldsymbol{\sigma} \times \boldsymbol{\sigma}_{N}\right] \cdot[\mathbf{q} \times \mathbf{k}]\left[\boldsymbol{\tau}-\boldsymbol{\tau}_{N}\right]_{z} / m_{p}^{2} .
\end{array}
$$

Here $\sigma\left(\sigma_{N}\right)$ is the Pauli matrix acting on the spin state of the antiproton (nucleon $N=p, n$ ) and $\tau$ $\left(\boldsymbol{\tau}_{N}\right)$ is the isospin matrix acting on the isospin state of the antiproton (nucleon). The momenta $\mathbf{q}$ and k were already defined above in the context of Eq. (2). Both terms in Eq. (3), $h_{N}$ and $g_{N}$, occur in the TVPC $p n$ interaction. The TVPC $p N$ scattering amplitude contains also the charge-exchange term

$$
t^{c h}=g_{N}^{\prime}\left(\boldsymbol{\sigma}-\boldsymbol{\sigma}_{N}\right) \cdot i[\mathbf{q} \times \mathbf{k}]\left[\boldsymbol{\tau} \times \boldsymbol{\tau}_{N}\right]_{z} / m_{p}^{2},
$$

which describes the elastic transitions $p n \rightarrow n p$ and $n p \rightarrow p n$. Within a picture of one-mesonexchange interaction this $g^{\prime}$-term corresponds to the charged $\rho$-meson exchange [19]. The same term (4) corresponds to the charge-exchange processes $\bar{p} p \rightarrow \bar{n} n$ or $\bar{n} n \rightarrow \bar{p} p$. However, in contrast to $p n$ scattering these processes are inelastic and therefore the operation of time-reversal invariance transforms, for example, the $\bar{p} p \rightarrow \bar{n} n$ amplitude to the $\bar{n} n \rightarrow \bar{p} p$ amplitude and does not impose any restrictions on these amplitudes.

The $h_{N}$-term in Eq. (3) can be associated with the axial $h_{1}$-meson exchange. As shown in Ref. [19], contributions of the $\pi$ - and $\sigma$-meson to the TVPC $N N$ interaction are excluded, which is obviously true for the TVPC $\bar{N} N$ interaction as well.

\subsection{TVPC amplitude of $\bar{p} d$ forward scattering}

One can write the $\bar{p} d$ forward elastic scattering amplitude in general form taking into account the TVPC $\bar{N} N$ interactions, as it was done for $p d$ elastic scattering [7, 17], and then apply the generalized optical theorem to derive Eq. (1) for the total $\bar{p} d$ scattering cross section. As in Ref. [7], the integrated cross section $\widetilde{\sigma}$ is related to the TVPC term $\widetilde{g}$ of the $\bar{p} d$ forward elastic scattering amplitude by $\widetilde{\sigma}=-4 \sqrt{\pi} \operatorname{Im} \frac{2}{3} \widetilde{g}$. Furthermore, the TVPC forward amplitude of $\bar{p} d$ elastic scattering $\widetilde{g}$ can be found within the Glauber theory [7]. We consider the $h_{N^{-}}$and $g_{N^{-}}$-terms and take into account both the $S$ and $D$-wave components of the deuteron. Taking into account that the $g_{N}$-term is excluded in the process $\bar{p} n \rightarrow \bar{p} n$ due to the isospin operator in Eq. (3), we obtain the following result for the TVPC forward amplitude from the corresponding equation in Ref. [11]:

$$
\begin{array}{r}
\widetilde{g}=\frac{i}{4 \pi m_{p}} \int_{0}^{\infty} d q q^{2}\left[S_{0}^{(0)}(q)-\sqrt{8} S_{2}^{(1)}(q)-4 S_{0}^{(2)}(q)+\sqrt{2} \frac{4}{3} S_{2}^{(2)}(q)+9 S_{1}^{(2)}(q)\right] \\
{\left[-C_{n}^{\prime}(q)\left(h_{p}+g_{p}\right)-C_{p}^{\prime}(q) h_{n}\right] .}
\end{array}
$$

Here $S_{i}^{(j)}$ are the elastic form factors of the deuteron defined in Ref. [11]. The first term in the (big) squared brackets in Eq. (5), $S_{0}^{(0)}(q)$, corresponds to the $S$-wave approximation, the second term, $S_{2}^{(1)}(q)$, accounts for the $S-D$ interference, and the last three terms contain the pure $D$-wave contributions. 


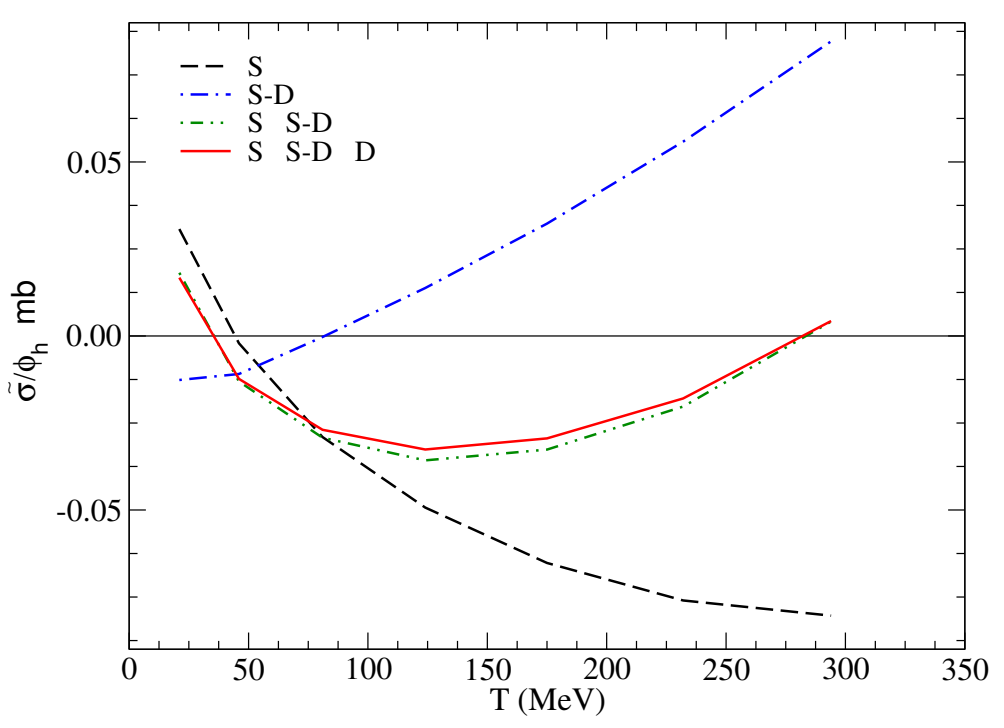

Figure 1. The TVPC signal $\widetilde{\sigma}$ for the $h$-term, in units of the ratio $\left(\phi_{h}\right.$, see Ref. [7]) of the TVPC and the strong $h_{1} N N$ coupling constants, versus the antiproton beam energy $T$. Results of our calculations accounting for different terms of the deuteron wave function in Eq. (5) are shown, based on the deuteron wave function of the CD Bonn potential and the hadronic $\bar{p} N$ amplitudes from Ref. [15]: $S$-wave (black), $S$ - $D$ interference (blue), $S+S-D$ waves (green), full result (red).

As was shown in Ref. [11], the contribution of the $g^{\prime}$-term to the null-test signal vanishes in $p d$ scattering due to the specific spin-isospin structure of the $g^{\prime}$-interaction. Formally, for the same reason the charge-exchange $g^{\prime}$-term given by Eq. (4) vanishes in the $\bar{p} d$ forward elastic scattering amplitude.

In the first theoretical work [5] where the null-test signal was calculated within the impulse approximation, the Coulomb interaction was not considered. In Ref. [6] Faddeev calculations were performed, but only for $n d$ scattering and at rather low energies of $\sim 100 \mathrm{keV}$. The Coulomb interaction was taken into account for the first time in Ref. [7] in a calculation of the null-test signal of $p d$ scattering within Glauber theory and found to be negligible. A similar result was found in Ref. [20] using Faddeev calculations.

\subsection{Numerical results}

Results of numerical calculations of the energy dependence of the null test-signal for the $h$-term are presented in Fig. 1, in units of the unknown TVPC coupling strength. One can see from this figure that the deuteron $S$-wave contribution (dashed line) leads to a smooth energy dependence and has a node at an antiproton beam energy of about $50 \mathrm{MeV}$. The inclusion of the $D$-wave changes this behaviour considerably (solid line) due to a destructive $S-D$ interference (cf. dash-dotted line). As a result, a second zero of the null-test signal $\widetilde{\sigma}$ appears at higher energies, i.e. at $T \approx 300 \mathrm{MeV}$. The maximal value of $\widetilde{\sigma}$ is expected at $100-150 \mathrm{MeV}$. Note that the actual position of the nodes changes only slightly when deuteron wave functions from other $N N$ models are used for the calculation.

Let us consider possible spurious effects that could mimic a TVPC signal. One source for a spurious signal is associated with a nonzero deuteron vector polarization $p_{d}^{y} \neq 0$ (in the direction of the incident-proton-beam polarization $\mathbf{p}^{p}$ ). In this case, the term $\sigma_{1} P_{y}^{\bar{p}} p_{y}^{d}$ in Eq. (1) contributes to the 
asymmetry corresponding to the difference of the event counting rates for the cases of $p_{y}^{\bar{p}} P_{x z}>0$ and $p_{y}^{p} P_{x z}<0$ (with the fixed sign of $P_{x z}$ ), which is planned to be measured at COSY [3]. According to our calculations, the integrated cross section $\sigma_{1}$ could be equal to zero at antiproton beam energies of $\sim 100 \mathrm{MeV}$ (see results for the Jülich $\bar{N} N$ interaction model in Refs. [21, 22]). Therefore, at this energy the spurios signal caused by a nonzero value of the deuteron vector polariziation $p_{y}^{d}$ could be minimized.

\section{Concluding remarks}

We have performed a study of time-reversal-invariance violating but parity conserving effects in antiproton-deuteron scattering. Specifically, we have evaluated the null-test TVPC signal for scattering of antiprotons with transversal polarization $p_{y}^{p}$ on deuterons with tensor polarization $P_{x z}$ on the basis of the spin-dependent Glauber theory. The observed effects turned out to be similar to those in $p d$ scattering: (i) There is a strong impact of the deuteron $D$-wave on the null-test signal that arises from a destructive interference between the $S$ - and $D$-wave contributions; (ii) There is an oscillating behaviour of the null-test signal as a function of the beam energy. Accordingly, it is possible that the signal for TVPC effects is zero at some specific energies, even when the TVPC interaction itself is nonzero.

Acknowledgement. This work was supported in part by the Heisenberg-Landau program.

\section{References}

[1] A.D. Sakharov, Pis'ma v ZHETF 5, 32 (1967).

[2] R.S. Conti, I.B. Khriplovich, Phys. Rev. Lett. 68, 3262 (1992).

[3] COSY Proposal N 215, Spokespersons: P.D. Eversheim, B. Lorentz, and Yu. Valdau, http://apps.fz-juelich.de/pax/paxwiki/images/8/8c/215-TRI_Prop_sum.pdf

[4] H.E. Conzett, Phys. Rev. C 48, 423 (1993).

[5] M. Beyer, Nucl. Phys. A 560, 895 (1993).

[6] Y.-H. Song, R. Lazauskas, V. Gudkov, Phys. Rev. C 84, 025501 (2011).

[7] Yu.N. Uzikov, A.A. Temerbayev, Phys. Rev. C 92, 014002 (2015).

[8] Yu. N. Uzikov, J. Phys. Conf. Ser. 678, 012020 (2016).

[9] Yu.N. Uzikov, EPJ Web Conf. 113, 04027 (2016).

[10] Yu.N. Uzikov, A.A. Temerbayev, Int. J. Mod. Phys. Conf. Ser. 40, 1660080 (2016).

[11] Yu.N. Uzikov, J. Haidenbauer, Phys. Rev. C 94, 035501 (2016).

[12] Yu.N. Uzikov, EPJ Web Conf. 138, 08001 (2017).

[13] A.A. Temerbayev, Yu. N. Uzikov, Izv. Ross. Akad. Nauk Ser. Fiz. 79, 535 (2015) [ Bull. Russ. Acad. Sci. Phys. 79, 493 (2015)].

[14] A.A. Temerbayev, Yu. N. Uzikov Izv. Ross. Akad. Nauk Ser. Fiz. 80, 271 (2016) [ Bull. Russ. Acad. Sci. Phys. 80 N4, 242 (2016)].

[15] D. Zhou, R.G.E. Timmermans, Phys. Rev. C 86, 044003 (2012).

[16] M.N. Platonova, V.I. Kukulin, Phys. Rev. C 81, 014004 (2010).

[17] A.A. Temerbayev, Yu.N. Uzikov, Yad. Fiz. 78, 38 (2015) [Phys. At. Nucl. 78, 35 (2015)].

[18] P. Herczeg, Nucl. Phys. 75, 655 (1966).

[19] M. Simonius, Phys. Lett. B 78, 147 (1975).

[20] Y.-H. Song, R. Lazauskas, V. Gudkov, Phys. Rev. C 93, 065501 (2016). 
[21] Yu. N. Uzikov, J. Haidenbauer, Phys. Rev. C 87, 054003 (2013).

[22] Yu. N. Uzikov, J. Haidenbauer, Phys. Rev. C 88, 027001 (2013). 